Bio - grafia. Escritos sobre la Biología y su Enseñanza. ISSN 2027

Edición Extraordinaria. p.p. 424 - 436

Memorias del VIII Encuentro Nacional de Experiencias en Enseñanza de la Biología y la Educación Ambiental. III Congreso Nacional de Investigación en Enseñanza de la Biología.

\title{
"¿CONSUMIR O NO ALIMENTOS MODIFICADOS GENÉTICAMENTE?, UNA CONTROVERSIDA SOCIENTIFÍCA PARA CONTRIBUIR A LA FORMACIÓN SCOIOPOLÍTICA EN LA CLASE DE CIENCIAS"
}

\author{
Cano, Daniel ${ }^{1}$; Echeverri, Eddy ${ }^{2} \&$ Giraldo, Paula ${ }^{3}$.
}

\begin{abstract}
${ }^{1}$ leinad9863@yahoo.com²eddyeche_017@hotmail.com³ paula.agm_74@hotmail.com
Universidad de Antioquia- Licenciatura en educación básica con énfasis en Ciencias Naturales y educación ambiental.
\end{abstract}

\section{RESUMEN}

Esta investigación tiene como propósito, contribuir a la formación sociopolítica de los estudiantes a partir de una propuesta pedagógica, en la cual se incentivan discusiones y debates sobre la producción y el consumo de alimentos modificados genéticamente. Dicho estudio atiende al llamado de Derek Hodson (En sus textos (2003, 2004 y 2010) en el cual se atiende el hecho de ir más allá del enfoque Ciencia Tecnología Sociedad, para formar científica y críticamente a la ciudadanía, a partir de un currículo que tenga en cuenta siete áreas de preocupación, en este caso con la controversia sociocientífica que se abordo, toma en cuenta dos áreas que son: - salud humana; alimentación y agricultura-.

El estudio se realizó con un grupo de estudiantes del grado octavo de la Institución Educativa Comercial Antonio Roldan Betancur, del municipio de Bello, Antioquia; esta propuesta de formación busca alejarse de la enseñanza tradicional de la ciencia para promover la formación de ciudadanos críticos e informados. 
Bio - grafia. Escritos sobre la Biología y su Enseñanza. ISSN 2027

Edición Extraordinaria. p.p. 424 - 436

Memorias del VIII Encuentro Nacional de Experiencias en Enseñanza de la Biología y la Educación Ambiental. III Congreso Nacional de Investigación en Enseñanza de la Biología.

La metodología de investigación acoge el paradigma cualitativo cuyo método elegido es el estudio de caso instrumental (Stake, 1998). Para analizar la información registrada durante las actividades se retomó la estrategia análisis de contenido (Piñuel, 2002), se

eligieron los enunciados construidos por los estudiantes como unidad de análisis y se extrajo de las actividades donde surgen dichas aseveraciones la unidad de contexto.

Se plantearon tres categorías de análisis con sus respectivas subcategorías que fueron diseñadas a la luz de los referentes teóricos y con base en los objetivos de investigación propuestos, dichas categorías se relacionan con el uso de conceptos científicos, reflexiones sobre la naturaleza de la ciencia y llamados a la acción sociopolíticas en posturas y puntos de vista de las estudiantes. Gracias a estos enunciados se permitirá construir conclusiones de como una propuesta pedagógica puede permitir la argumentación, la crítica y generar acciones en estudiantes a través de las discusiones generadas por los organismos genéticamente modificados en la clase de ciencias naturales.

\title{
PALABRAS CLAVES
}

Formación sociopolíticas, organismos genéticamente modificado, transgénicos.

\begin{abstract}
This research aims to contribute to the socio-political education of students from a pedagogical proposal, which discussions and debates on the production and consumption of genetically modified foods are encouraged. This study serves to call Derek Hodson (In his writings (2003, 2004 and 2010) calls for going beyond the scope Science and Technology
\end{abstract}


Bio - grafia. Escritos sobre la Biología y su Enseñanza. ISSN 2027

Edición Extraordinaria. p.p. 424 - 436

Memorias del VIII Encuentro Nacional de Experiencias en Enseñanza de la Biología y la Educación Ambiental. III Congreso Nacional de Investigación en Enseñanza de la Biología.

Society proposes a curriculum for sociopolitical training includes the study of sociopolitical issues) to form scientific and critically citizens from a curriculum that takes into account seven areas of concern, in this case with the social scientific controversy addressed two areas: - human health; food and agriculture.

The study was conducted with a group of eighth grade students of School Business Antonio Roldan Betancur, the municipality of Bello, Antioquia;This training proposal seeks to move away from traditional teaching of science to promote the formation of critical and informed citizens. The research methodology qualitative paradigm which hosts the chosen method is instrumental case study as a strategy to analyze the information recorded during the activities we return to the content analysis which takes into account the statements constructed by students as analysis unit activity where such claims arise as context unit.

Analysis three categories and their respective subcategories were designed in the light of the theoretical framework and the basis of the proposed research objectives were raised these categories are related to the use of scientific concepts, reflections on the nature of science and called to Action Socio-political positions and views of the students. Thanks to these statements is allowed to build conclusions as a pedagogical approach can allow the argument, criticism and generate action on students through discussions generated by generically modified organisms.

KEYWORDS: Sociopolitical formation, genetically modified organisms, transgenics. 
Bio - grafia. Escritos sobre la Biología y su Enseñanza. ISSN 2027

Edición Extraordinaria. p.p. 424 - 436

Memorias del VIII Encuentro Nacional de Experiencias en Enseñanza de la Biología y la Educación Ambiental. III Congreso Nacional de Investigación en Enseñanza de la Biología.

\section{INTRODUCCIÓN}

Partiendo del hecho que la enseñanza de las ciencias, por lo general es dado de manera memorística, ahistórica y descontextualizada (Hodson, 2003) surge la necesidad y preocupación por parte de la comunidad docente de buscar alternativas para lograr desde su ejercicio profesional, que los estudiantes comprendan, se incentiven y desarrollen habilidades que mediante la argumentación y la toma de decisiones, permita ser partícipes activos en discusiones públicas sobre cuestiones de Ciencia y Tecnología, y promoviendo la formación de ciudadanos autónomos y responsables (Solbes y Travers, 1996).

De esta manera, para la elaboración de propuestas que pretendan mejorar la práctica docente y el aprendizaje, es necesario el análisis de lo que se enseña, cómo se enseña y de las condiciones que se generan en la enseñanza habitual para el aprendizaje de las ciencias. Teniendo en cuenta lo anterior, la enseñanza de las ciencias puede ser tomada a partir de la problematización de esta misma, que como lo plantea Hodson es necesario que esto se refleje en un currículo que permita a los estudiantes hacer ciencia, aprender ciencia y escribir sobre la ciencia (Hodson, 2003).

Una forma de problematizar la ciencia es implementar los asuntos sociocientíficos -ASC- y la argumentación, los primeros hacen referencia a debates, polémicas, dilemas y controversias sociales generadas por conceptos, productos, procedimientos y técnicas que proceden de las ciencias (Kolstø, 2000). En cuanto a la argumentación esta permite mejorar del razonamiento cognitivo; el desarrollo de la compresión conceptual; el incremento de la comprensión de los estudiantes acerca de la naturaleza epistémica de la ciencia; y la 
Bio - grafia. Escritos sobre la Biología y su Enseñanza. ISSN 2027

Edición Extraordinaria. p.p. 424 - 436

Memorias del VIII Encuentro Nacional de Experiencias en Enseñanza de la Biología y la Educación Ambiental. III Congreso Nacional de Investigación en Enseñanza de la Biología.

proporción de una experiencia afectiva que es tanto positiva como atractiva (Osborne, 2003).

En este sentido, la implementación de estrategias basadas en el trabajo con cuestiones sociocientíficas fomentan habilidades como la argumentación, la toma de decisiones y el pensamiento crítico, por medio de la participación en debates o intervenciones (escritas u orales) generadas a través del impacto de la ciencia y la tecnología en aspectos sociales (Zeidler, et al, 2003)

Así pues, con el fin de alejarnos de la enseñanza tradicional y permitir la formación sociopolítica, a través de la enseñanza de las ciencias, se plantea la siguiente propuesta pedagogíca: "la alimentación: entre lo orgánico y lo transgénico, aportes a una nutrición saludable", como un ASC, que busca la transversalidad $e$ interdisciplinariedad de los conocimientos científicos en el aula y en el aprendizaje de la ciencia. Se plantea una secuencia de actividades en términos del tópico; "alimentos transgénicos y alimentos orgánicos" como tema controversial, abordando en las implicaciones que han vivido en el país, viéndolo desde una perspectiva social, económica, científica y ambiental a fin de posibilitar una reflexión crítica en el aula por parte tanto de los estudiantes como del docente.

La elección del ASC alimemtos geneticamente modificados, se debe a que es una situación asociada con la alimentación y nutrición de las personas, convirtiéndose en un tema cercano a la cotidianidad del estudiante; de igual modo se basa en los lineamientos establecidos por el Ministerio de Educación Nacional, al trabajar el tema de nutrición, y de genética y sus 
Bio - grafia. Escritos sobre la Biología y su Enseñanza. ISSN 2027

Edición Extraordinaria. p.p. 424 - 436

Memorias del VIII Encuentro Nacional de Experiencias en Enseñanza de la Biología y la Educación Ambiental. III Congreso Nacional de Investigación en Enseñanza de la Biología.

implicaciones en el grado Octavo. Resaltando que muchas veces la atención en esas temáticas es mínima, mencionándose de manera superficial, sin dar mayor énfasis e importancia.

Razón por la cual, se propone la implementación de unas actividades con el fin de lograr mediante la integración de contenidos, el aumento en los niveles de argumentación y el desarrollo de las capacidades de los estudiantes, para resolver situaciones cotidianas de interés.

\section{METODOLOGÍA}

La investigación está estructurada bajo el paradigma cualitativo, porque se busca el reconocimiento y la comprensión de una situación que se quiere llevar al aula de clase y cuál es su consecuencia al implementarla, por lo cual se quiere desarrollar la descripción de un hecho particular y siguiendo un determinado proceso hasta llegar a una generalización y además bajo esta perspectiva se permite la interacción entre los estudiantes y profesores por lo cual se favorece el proceso de argumentación (Stake, 1998).

Teniendo en cuenta el paradigma cualitativo y en vista de los objetivo pactados en un principio de la propuesta, se escoge como tipo de investigación cualitativa el estudio de caso porque es la más apropiada a nuestro objeto de estudio ya que se espera que se abarque la complejidad de un caso particular (Stake,1998), en este caso la formación sociopolítica de los estudiantes a partir de la implementación de asuntos sociocientíficos, utilizando dilemas relacionados con la alimentación saludable y el consumo o no de los organismos genéticamente modificados. 
Bio - grafia. Escritos sobre la Biología y su Enseñanza. ISSN 2027

Edición Extraordinaria. p.p. 424 - 436

Memorias del VIII Encuentro Nacional de Experiencias en Enseñanza de la Biología y la Educación Ambiental. III Congreso Nacional de Investigación en Enseñanza de la Biología.

Puesto que las intervenciones que se han realizado, y que las actividad que se han realizado han sido dentro del aula de clase, se opta por realizar análisis de tipo instrumental donde el toman con la misma importancia las interacciones dentro del aula de clase. (Stake, 1998).

Las intervenciones en el grado octavo, en el cual solo había mujeres a causa del modelo pedagógico diferencial que se aplica en la institución, se realizó por medio de una propuesta pedagógica la cual constaba de diversas actividades como la implementación de un cuestionario, videoforo, juego de rol, la ruta de interrogantes, la realización de una carta y la fomentación por medio de una feria. Dichas actividades, tuvieron como objetivo proyectar los asuntos sociocientíficos como estrategia de la enseñanza para las ciencias naturales, además fomentar la formación sociopolítica en el aula de clase, ya que desde las actividades propuesta desde la propuesta, las estudiantes debieron asumir una posición frente a un dilema atravesado por diferentes dimensiones como la ética, ambiental, política y moral, y al incentivar la toma de decisiones y posiciones se está contribuyendo a que el estudiante se motive a participar y desempeñar acciones de acuerdo a su posición. Al mismo tiempo cada actividad, estará sustentada por la argumentación y tendrá como finalidad promover acciones, pensamiento crítico y reflexiones, en las estudiantes. Para terminar de especificar la metodología se hace importante resaltar que en el colegio se interesa en proyectos relacionados con agricultura y agropecuaria por el cual el tema de la alimentación saludable enfocado en los alimentos orgánicos y en los que son genéticamente modificados, son de gran importancia para la tranversalizar el currículo con los proyectos institucionales. 
Bio - grafia. Escritos sobre la Biología y su Enseñanza. ISSN 2027

Edición Extraordinaria. p.p. 424 - 436

Memorias del VIII Encuentro Nacional de Experiencias en Enseñanza de la Biología y la Educación Ambiental. III Congreso Nacional de Investigación en Enseñanza de la Biología.

Siguiendo las características que se expone desde la metodología de estudio de caso, se infiere que la mejor manera de tratar el análisis e interpretación de los enunciados que sobresalen como producto de esta propuesta investigativa va ser por medio del análisis de contenido tal como lo expone Jose Luis Piñuel (2002), en el cual es entendido como un conjunto de procedimientos interpretativos de productos comunicativos tales como mensajes o textos escritos, y discursos orales que se dan dentro del aula, donde el análisis interpretativo se centra en los contenidos propios que conforma el producto comunicativo y no la forma lingüística en la que se comunica.

\section{Categorías de análisis, propuesta pedagógica y protocolo ético.}

Teniendo en cuenta el paradigma, metodología de investigación escogida y los objetivos pactados, se elaboró una propuesta pedagógica la cual conto con una serie de instrumentos que hicieron posible el registro de la información y posteriormente hacer el análisis y el tratamiento de esta a través de unas categorías para la debida interpretación, por lo cual se planteó la siguiente ruta de actividades, que actualmente se realizaron exceptuando la actividad de la feria donde se expone las ideas de acciones frente al asunto en el que se ha venido trabajando. Teniendo en cuanta que los aportes que se tomaron de la propuesta pedagógica por parte de los estudiantes van ser divulgados y analizados, se hace el proceso de protocolo ético donde se aclara que la información que ellas expongan durante el proceso solo será utilizada con fines académicos:

La propuesta que se realizó para el registro de información utilizada posteriormente par la investigación tomo el siguiente nombre: 
Bio - grafia. Escritos sobre la Biología y su Enseñanza. ISSN 2027

Edición Extraordinaria. p.p. 424 - 436

Memorias del VIII Encuentro Nacional de Experiencias en Enseñanza de la Biología y la Educación Ambiental. III Congreso Nacional de Investigación en Enseñanza de la Biología.

\section{"LA ALIMENTACIÓN: ENTRE LO ORGÁNICO Y LO TRANSGÉNICO, APORTES A UNA NUTRICIÓN SALUDABLE", COMO UNA PROPUESTA DE FORMACIÓN SOCIPOLOLITICA EN LA CLASE DE CIENCIAS"}

En todas las actividades que se realizaron se notó el interés y la motivación por parte de los estudiantes, también es importante hacer notar que siempre se utilizaron herramientas como video, e imágenes las cuales tuvieron buena acogida por parte de loes estudiantes.

\section{SECUENCIA DE ACTIVIDADES}

Actividad 1 Cuestionario de indagación: un cuestionario que permitirá reconocer que tipos de alimentos consumen las estudiantes y si reconocen los transgénicos. Actividad 2 Video Foro: a través de una serie de videos (a favor y en contra de los transgénicos), dará cabida a identificar algunas opiniones de las estudiantes por la temática de los organismos genéticamente modificados.

Actividad 3 Ruta de interrogantes: un recorrido por diferentes bases, donde las estudiantes se apoyaron en noticias y en las consultas realizadas, para que respondieran a las 'preguntas quue se enfrentaban en cada base. Las estaciones que se elaboraron fueron las siguientes: (1) Monsanto según Monsanto, donde se muestra la perspectiva de la multinacional Monsanto se proyecta ante la sociedad, su finalidad comercial y lo que pretenden como compañía, (2) Organismos modificados genéticamente (animales y vegetales), donde a través de imágenes (pollos sin plumas, vacas musculosas, cerdos $\sin$ huesos y un tomate grande y de color opaco) podrán discutir acerca de con que 
Bio - grafia. Escritos sobre la Biología y su Enseñanza. ISSN 2027

Edición Extraordinaria. p.p. 424 - 436

Memorias del VIII Encuentro Nacional de Experiencias en Enseñanza de la Biología y la Educación Ambiental. III Congreso Nacional de Investigación en Enseñanza de la Biología.

intencionalidad modifican genéticamente a los organismos y reflexionar sobre las consecuencias finales en los organismos, (3) Bioética, en esta sección dialogarán sobre la ética que se evidencia en esta problemática, además de asumir que harían si estuvieran en cierto caso (ya sean que sean comerciantes, científicas o incluso el mismo animal (¿qué pasaría si te quitan las plumas, siendo un pollo? O ¿si te quitan el abrigo en el polo norte?), (4) Franquicias y multinacionales, donde leerán noticias sobre algunos productos que se han evidenciado que utilizan O.G.M, también observando video de comerciales que han salido en televisión nacional sobre el consumo de ciertos productos (Como: A comer pollo, carne de cerdo), para así analizar con que fin se hace y discutir sobre el consumo, (5) Carta, esta será una propuesta donde escribirán a un estamento gubernamental o al presidente, que piensan al respecto de los O.G.M, resaltando lo anterior (en las otras bases) analizado, así mismo proponer posibles propuestas contra el asunto. Actividad 4 Panel de expertos: es un juego de rol, en donde seis estudiantes representaran a una ambientalista, una científica y una campesina (roles en contra de los $O G M)$, mientras que una ingeniera genética, una política y una empresaria, defenderán los buenos de los

OGM.

Actividad 5 Feria: por medio de una feria las estudiantes mostraran propuestas propias para la comunidad educativa, donde reflejen sus posturas sobre los OGM. Consta de varios stands donde el resto de la comunidad de la institución podrá visitarlos para escuchar y/o leer, lo que las estudiantes proponen. Como discusiones, carteles, entre otros. 
Bio - grafia. Escritos sobre la Biología y su Enseñanza. ISSN 2027

Edición Extraordinaria. p.p. 424 - 436

Memorias del VIII Encuentro Nacional de Experiencias en Enseñanza de la Biología y la Educación Ambiental. III Congreso Nacional de Investigación en Enseñanza de la Biología.

Atendiendo las características del análisis que se quiere implementar para cumplir los objetivos establecidos, las categorías de análisis las cuales se abrirán para la interpretación adecuada de los enunciados de las estudiantes serán las siguientes: Conceptos en ciencias naturales, Naturaleza de la ciencia (subdividida en: Limites de la ciencia y Valores de la ciencia) y Acciones

A pesar de que el proyecto investigativo no se ha terminado se han registrados enunciados que se han ubicado en todas las categorías de análisis especificadas anteriormente, que consideramos que son muy oportunas para el análisis y también da luz al cumplimiento de los objetivos investigativos.

\section{REFERENCIAS BIBLIOGRÁFICAS}

Aikenhead, G. S. (2005). Research into STS Science Education. Educación Química, 16(3), pp.

Arnal, J.; Del Rincón, D. Latorre, A. \& (1996) Bases metodológicas de la investigación educativa. Barcelona: 92

Carrión, D. Castro, P. (2012). Y tú, ¿qué tipo de carne prefieres en tu hamburguesa?, una cuestión sociocientífica que promueve la argumentación. Segunda parte: memorias ler foro. Universidad pedagógica. MaDoQuim: memorias de la maestría en docencia de la química. $\mathrm{N}^{\circ} 2$.

Hodson, D. (1994). Seeking Directions for Change. The Personalization and Politisation of Science Education. Curriculum Studies, 2(1), 71-98. 
Bio - grafia. Escritos sobre la Biología y su Enseñanza. ISSN 2027

Edición Extraordinaria. p.p. 424 - 436

Memorias del VIII Encuentro Nacional de Experiencias en Enseñanza de la Biología y la Educación Ambiental. III Congreso Nacional de Investigación en Enseñanza de la Biología.

Hodson, D. (2003). Time for action: Science education for an alternative future. International Journal of Science Education, 25, pp. 645-670.

Hodson, D (2004). Going Beyond STS: Towards a Curriculum for Sociopolitical

Jiménez- Aleixandre (2010) "Capacidad de desarrollar uan opinion independiente adquiriendo la facultad de reflexionar sobre la sociedad y participar en ella" (Generar acciones y comprender el ASC).

Kolstø, S.D. (2000). Consensus projects: Teaching science for citizenship. International Journal of Science Education, 22(6), 645-664.

Medina, L. Lambraño, D. Villamizar, D (2012). Unidad didáctica: calimentos transgénicos, la respuesta a una problemática ambiental o una estrategia económica de las grandes superpotencias. MaDoQuim: memorias de la maestria en docencia de la quimica. N²

Osborme, J (2009). Hacia una pedagogía más social en la educación científica: el papel de la $\begin{array}{lll}\text { argumentación. } \quad \text { Educ. 20(2), } & \end{array}$ $145-154$

Piñuel, J. L. (2002) Epistemología, metodología técnicas de análisis de contenido. Estudios de sociolingüística. 3 (1), 1 -42 Madrid: Universidad Complutense de Madrid.

Solbes \& Traver (1996) la enseñanza de las ciencias ha contribuido al dogmatismo, el formulismo, el ahistoricismo y a la decontextualización (ausencia de relaciones CTS)

Stake, R. E. (1998) Investigar con estudios de caso. Madrid: Morata. 
Bio - grafia. Escritos sobre la Biología y su Enseñanza. ISSN 2027

Edición Extraordinaria. p.p. 424 - 436

Memorias del VIII Encuentro Nacional de Experiencias en Enseñanza de la Biología y la Educación Ambiental. III Congreso Nacional de Investigación en Enseñanza de la Biología.

Zeidler, D. L., Osborne, J, Erduran, S., Simon, S. \& Monk, M. (2003). The role of argument during discourse about socioscientific issues. En D.L. Zeidler (ed.), The Role of Moral Reasoning on Socioscientific Issues and Discourse in Science Education 